\title{
Parameter Design for Signal-Response Systems: A Different Look at Taguchi's Dynamic Parameter Design
}

\author{
Arden Miller and C. F. J. Wu
}

\begin{abstract}
A recent trend in the industrial applications of robust parameter design is to consider complex systems which are called "systems with dynamic characteristics" in Taguchi's terminology or signalresponse systems in this paper. This potentially important tool in quality engineering lacks a solid basis on which to build a rigorous body of theory and methodology. The purpose of this paper is to provide such a basis. We classify signal-response systems into two broad types: measurement systems and multiple target systems. Three issues are then of fundamental importance. First, a proper performance measure needs to be chosen for system optimization, and this choice depends on the type of system. Taguchi's dynamic signal-to-noise ratio is shown to be appropriate for certain measurement systems but not for multiple target systems. Second, there are two strategies for modeling and analyzing data: performance measure modeling and response function modeling. Finally, the proper design of such experiments should take into account the modeling and analysis strategy. The proposed methodology is illustrated with a real experiment on injection molding.
\end{abstract}

Key words and phrases: Dynamic SN ratio, measurement systems, multiple target systems, robust parameter design, performance measures, response function modeling.

\section{INTRODUCTION}

Taguchi (1987) introduced the robust parameter design (RPD) methodology as a method of improving the quality of a process or product by making it less sensitive to factors which cause variability. There has been much discussion in the literature about his methodology and alternative procedures have been proposed. See Nair (1992) and Box (1988) for general discussions. Most of the statistical literature concerning robust parameter design involves situations where the quality characteristic of interest (response) is a single quantity, $Y$, which has a specified optimal value. For example, Shoe-

Arden Miller is Lecturer, Department of Statistics, University of Auckland, Private Bag 92019, Auckland 1, New Zealand. C. F. J. Wu is Professor, Department of Statistics and of Industrial and Operations Engineering, University of Michigan, Ann Arbor, Michigan 48109-1027. maker, Tsui and Wu (1991) presented a case study involving the plating of silicon layers on wafers. In this case, the response was the thickness of the silicon layer. A target value of 14.5 micrometers had been identified and the goal of the experiment was to determine conditions which would result in the distribution of $Y$ being as concentrated as possible around this value. Another example, presented by Pignatiello and Ramberg (1985), involves a heat treating process of leaf springs used on trucks. The objective was to develop a process which would result in springs whose free height was as close as possible to the target value of 8 inches. Taguchi used the somewhat confusing term "static characteristic" to refer to this type of application, which we call a simple response system.

Taguchi also identified a second type of application for robust parameter design methodology which he called "dynamic characteristics." Simply stated, this refers to situations where the response is required to assume different values as a result of 
changes in a signal factor, $M$. For example, a method for determining the amount of calcium in a water sample must produce different responses for different amounts of calcium. The performance of this type of system can only be evaluated by considering the relationship between the response and the actual amount of calcium present in samples (signal). As the term dynamic is misleading, we call this a signal-response system because the signal-response relationship is of prime importance. Since many engineering systems can be adequately described as signal-response systems, this methodology has become increasingly important in engineering applications. See, for example, many case studies in the American Supplier Institute Symposia on Taguchi Methods (Dearborn, Michigan). In spite of the practical impact, signal-response systems have received little attention in the research literature.

In this article we consider in detail an experiment described by DeMates (1990) on injection molding. As the system was required to inject different amounts of material for different applications, a reliable method of controlling the amount of material injected was needed. Part weight was adopted as the response, and high injection pressure was chosen as the signal factor due to its known ability to change the amount of material injected. Seven control factors, each at two levels, were included in the experiment (see Table 1). The term control factors designates factors which can be readily adjusted to different levels by the process operator and once set remain constant during the operation of the system. For the injection molding experiment, the control factors were chosen since they were thought to have the potential of affecting variability in part weight. One compound noise factor which represents the settings of four confounded noise factors was used (see Table 2). A noise factor is a factor which may vary during the operation of the system (and thus contribute to variability in the response) but can be held constant for the purposes of an experiment. Noise factors are often included in RPD experiments to insure that important sources of variability are investigated since the traditional approach of using replicate observations to assess variability is often ineffective. Replication assumes that the observations reflect the variability in the entire population of interest which is seldom reasonable in practice as real systems tend to change over time. For example, key parts wear over time, operators change from shift to shift, batches of raw materials vary, machine settings may drift and climatic conditions vary over days as well as over weeks and months. Even supposing the sample is representative of the population, the number
TABLE 1

Control factors for injection molding case study

\begin{tabular}{lcc}
\hline \multicolumn{1}{c}{ Factor } & $X=+1$ & $X=-1$ \\
\hline A: injection speed & 2.0 & 0.0 \\
B: clamp time & $44 \mathrm{~s}$ & $49 \mathrm{~s}$ \\
C: high injection time & $6.3 \mathrm{~s}$ & $6.8 \mathrm{~s}$ \\
D: low injection time & $17 \mathrm{~s}$ & $20 \mathrm{~s}$ \\
E: clamp pressure & $1,900 \mathrm{psi}$ & $1,700 \mathrm{psi}$ \\
F: water cooling & $70^{\circ} \mathrm{F}$ & $80^{\circ} \mathrm{F}$ \\
G: low injection pressure & $650 \mathrm{psi}$ & $550 \mathrm{psi}$ \\
\hline
\end{tabular}

of replicates required to get a sufficiently precise estimate of variability will, in most cases, be too large to be economically feasible (see Gunter, 1988). Available knowledge of the system, observational studies, and screening experiments can be useful methods of identifying important noise factors. In some experiments compound noise factors are used to reduce the required number of runs (see Phadke, 1989). A discussion of the use of compound noise factors is contained in Section 6 .

This article discusses robust parameter design for signal-response systems using the injection molding experiment to illustrate the methodology. Two distinct types of signal-response systems, measurement systems and multiple target systems, are identified in Section 2. This classification plays an important role in our methodological development. For example, the choice of the performance measure used for parameter design optimization depends on the type of system (see Section 3). It turns out that Taguchi's dynamic signal-to-noise (SN) ratio, see (2), is justifiable for many measurement systems but not for multiple target systems. Two strategies for modeling and analyzing data, performance measure modeling and response function modeling, are discussed in Section 4. The former includes Taguchi's SN ratio analysis as a special case, while the latter is a more flexible approach which can be tailored for each type of system. Both strategies are illustrated using the injection molding experiment in Section 5. Strategies for designing experiments

TABLE 2

Compound noise factor for injection molding case study

\begin{tabular}{lll}
\hline Label & \multicolumn{1}{c}{ Factor } & \multicolumn{1}{c}{ Levels } \\
\hline$X_{N}=+1$ & Melt index & 22 \\
& Percent regrind & $0 \%$ \\
& Operator & Experienced \\
& Resin moisture & Low \\
$X_{N}=-1$ & Melt index & 18 \\
& Percent regrind & $5 \%$ \\
& Operator & New \\
& Resin moisture & High \\
\hline
\end{tabular}


are discussed in Section 6 and concluding remarks are made in Section 7.

\section{CLASSIFICATION OF SIGNAL-RESPONSE SYSTEMS}

It is useful to classify signal-response systems according to the function of the system as this affects the manner in which the performance of the system should be evaluated. We have come across two common types of signal response systems (there may be others): multiple target systems and measurement systems.

A multiple target system is a system whose function requires that the value of a response quantity can be adjusted by changing the level of a signal factor. One example is the injection molding system described in Section 1. Another example can be found in Yano (1991, page 293). In this case, the quality characteristic of interest was the surface roughness of parts after the surface has been machined using a lathe. Since different applications required a different degree of surface roughness, some method of controlling the surface roughness of machined parts was needed. As experience indicated that the feed rate of the tool bit could effectively be used to alter the roughness of the machined surface, it was chosen as the signal factor. It was thought that factors such as type of lathe, cutting speed, depth of tool cut, type of tool cut, corner radius, cutting edge angle, front escape angle and side scoop angle may affect the relationship between feed rate and surface roughness. An experiment was conducted to select settings for these control factors which would allow surface roughness to be reliably controlled by the feed rate of the tool bit.

A measurement system is the process used to obtain an estimate of some quantity of interest for a given unit or sample. This may include sampling, sample preparation and calibration, as well as the actual measurement process. The true amount of the quantity present can be considered as an input signal $M$ which the system converts into a measured value or response $Y$. The precision with which $M$ can be estimated based on $Y$ is determined by the characteristics of the relationship between $M$ and $Y$. As an example, consider an experiment performed at a foundry to optimize an eddy current measurement procedure used to measure part hardness. An eddy current machine uses the intensity of feedback $(Y)$ from an electronic probe to estimate hardness $(M)$. Three control factors, (i) frequency, (ii) probe temperature and (iii) gain, were identified which represented factors that could be easily changed and were thought to affect the relationship between hardness and feedback intensity. Two noise factors, (i) part chemistry and (ii) part cleanliness, were included in the experiment since these represented conditions which changed from measurement to measurement and thus introduced variation into the process. In simple terms, the purpose of the experiment was to identify settings of the control factors which would make feedback intensity sensitive to changes in hardness but insensitive to changes in part chemistry and part cleanliness.

\section{PERFORMANCE MEASURES}

An important step in examining a signalresponse system is to identify a performance measure (PM) which evaluates the suitability of a given signal-response relationship for the intended application. By optimizing the chosen measure, control factor settings which achieve the desired engineering objectives can be identified.

One approach to identifying a suitable PM is to specify an ideal or target signal-response relationship and penalize for deviations from this target function. A good example of this approach is the PM Taguchi recommends for signal-response systems (see Phadke, 1989, page 114). Suppose the target function is of the form

$$
E(Y)=\beta_{t} M,
$$

where $\beta_{t}$ is the target slope and the actual signalresponse relationship can be represented by

(1) $Y=f(M)+\varepsilon, \quad$ where $\mathrm{E}(\varepsilon)=0, \mathrm{~V}(\varepsilon)=\sigma^{2}$.

A PM can then be generated by averaging the mean square error (MSE) over a specified range for the signal factor, say $\left(m_{a}, m_{b}\right)$,

$\mathrm{PM}=\int_{m_{a}}^{m_{b}} \mathrm{MSE} d m=\int_{m_{a}}^{m_{b}}\left(\left[f(m)-\beta_{t} m\right]^{2}+\sigma^{2}\right) d m$

In evaluating the performance of a system it may be beneficial to modify the PM if there exists a special type of control factor called an adjustment factor (see Leon, Shoemaker and Kacker, 1987, for an in-depth discussion of adjustment factors). In practical terms, an adjustment factor affects the system in a well-understood manner so that if the signalresponse function is known (or estimated) for any setting of the adjustment factor, then the signalresponse function can be reliably deduced for any other level of the adjustment factor. If such a factor exists, then it makes sense to evaluate the PM given the adjustment factor is set to its optimal level. Taguchi's dynamic SN ratio assumes the existence of an adjustment factor which affects the system in the same manner as a change of scale. Suppose for a fixed set of control factor levels we 


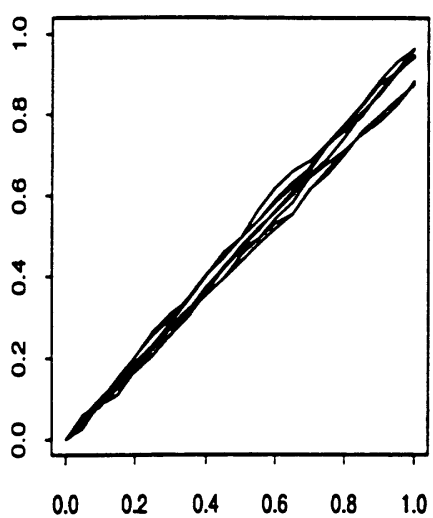

(a)

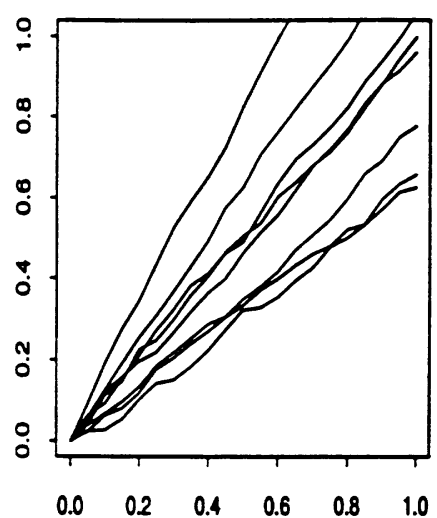

(b)

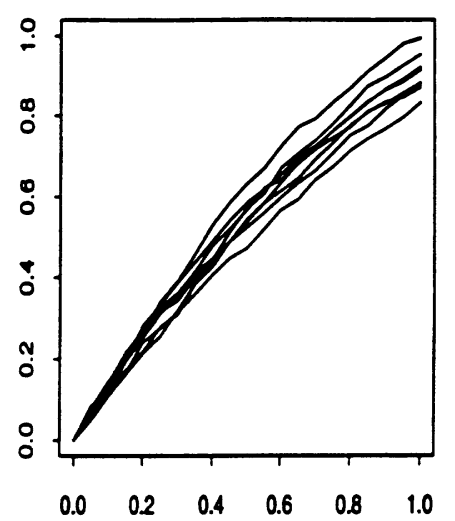

(c)

FIG. 1. Examples of signal-response relationships.

have the true signal-response relationship given in (1). Then by changing the adjustment factor we can obtain any signal-response relationship of the form $Y=c[f(M)+\varepsilon]$, where $c$ is any positive constant. To see how the adjustment factor is used in practice, suppose a set of observations are made for a fixed set of control factor levels, and let $y_{i j}$ represent the $j$ th observed response at the $i$ th signal level $\left(M_{i}\right)$. Now consider the least squares fit to the model

$$
E\left(y_{i j}\right)=\beta M_{i} .
$$

Let $\hat{\beta}$ represent the least squares estimate of $\beta$ and $s^{2}=(n-1)^{-1} \sum_{i} \sum_{j}\left(y_{i j}-\hat{\beta} M_{i}\right)^{2}$, where $n$ is the total number of observations. So $\hat{\beta}$ represents the estimated slope for the best fitting linear model and $s^{2}$ represents the estimated MSE for this model (averaged over the signal levels). If we assume the target function is $\beta_{t} M$, then we would wish to use the adjustment factor to scale the signal-response relationship by a factor of $\beta_{t} / \hat{\beta}$. Given this adjustment, the projected MSE would be $\left(\beta_{t} / \hat{\beta}\right)^{2} s^{2}$. Minimizing this MSE is equivalent to maximizing

$$
\log \left(\hat{\beta}^{2} / s^{2}\right)
$$

which Taguchi called the dynamic SN ratio. This PM is based on the objectives that the ideal signalresponse relationship should be (a) linear and (b) robust to uncontrolled factors. Figure 1 illustrates the difference between a good signal-response system (Figure 1a) and poor signal response systems (Figure 1b, c) according to this measure. Each line represents an observed signal-response curve for a given set of noise factor conditions. The first system is linear and relatively insensitive to noise factors whereas the second system is sensitive to noise and the third system is nonlinear.
Rather than developing a performance measure by identifying an ideal signal-response relationship and then penalizing departures from this ideal, we prefer to base performance measures directly on the ability of a system to perform its designated function. One reason for this is that it is not always possible to identify an ideal signal-response relationship. Consider a situation where the relationship is not linear in the original metrics of the response and signal factor but can be made linear by a suitable transformation of the signal factor. In this case, a PM based on a linear ideal function may well yield different values depending on whether the original or transformed metric is used for the signal factor. Another criticism of the dynamic SN ratio concerns its assumption of the existence of an adjustment factor which acts like a scaling factor which does not hold in many practical situations.

In the following subsections these performance measures will be developed separately for two types of systems: measurement systems and multiple target systems.

\subsection{Measurement Systems}

As the purpose of a measurement system is to obtain an estimate of some quantity of interest, it is reasonable that the system should be evaluated with respect to the precision of estimates obtained.

Mandel (1964, page 366) developed a "criterion for technical merit," as a method of comparing the relative merits of measurement systems. Mandel only considered systems where the variation of the response was constant across signal levels. Let $E(Y)=g(M)$ and $V(Y)=\sigma^{2}$, where $g$ is an invertible function with inverse $g^{-1}$. In this case, assuming $g(M)$ is known, the variance of $\hat{M}=g^{-1}\left(y_{\text {obs }}\right)$ 
can be approximated by $\sigma^{2} /\left[g^{\prime}(M)\right]^{2}$. Mandel defined $\sigma /\left|g^{\prime}(M)\right|$ as his criterion. Although Mandel only considered the comparison of different systems, his criterion could also be used to optimize a particular system.

Taguchi (1987, page 629) specifically considered the optimization of measurement systems which are based on linear calibration curves. Suppose $E(Y)=$ $\beta_{0}+\beta_{1} M$ and $V(Y)=\sigma^{2}$. Taguchi, in essence, adopted $\omega=\beta_{1}^{2} / \sigma^{2}$ as a performance measure (SN ratio), which he justified on the basis that it is the reciprocal of the estimation variance for $\hat{M}=\left(y_{\mathrm{obs}}-\right.$ $\left.\beta_{0}\right) / \beta_{1}$, provided $\beta_{0}, \beta_{1}$ and $\sigma^{2}$ are assumed known. Clearly, Taguchi's SN ratio is a special case of Mandel's criterion.

The measures developed by Taguchi and Mandel assume that the error variance is constant over the levels of the signal factor. There are applications which require this assumption to be relaxed. For example, Bocek and Novak (1970) discussed the use of gas chromatography for quantitative analysis and indicated that under certain circumstances this involves nonconstant variance. Suppose the system can be meaningfully represented by a model consisting of a location function and a variance function as follows:

$$
E(Y)=g(M), \quad V(Y)=h(M),
$$

where $g$ is a monotonic function of $M$. Then for an observed value of $Y=y_{\text {obs }}$, the classical estimator of $M$ is

$$
\hat{M}\left(y_{\text {obs }}\right)=g^{-1}\left(y_{\text {obs }}\right),
$$

where $g^{-1}$ is the inverse function of $g$. The variance of $\hat{M}$ is approximately

$$
\mathrm{V}\left(\hat{M} \mid y_{\mathrm{obs}}\right) \approx \frac{\mathrm{V}\left(Y \mid M=m_{t}\right)}{\left[g^{\prime}\left(m_{t}\right)\right]^{2}}=\frac{h\left(m_{t}\right)}{\left[g^{\prime}\left(m_{t}\right)\right]^{2}},
$$

where $g^{\prime}(M)=d g(M) / d M$ and $m_{t}$ is the true value of $M$ for the unit being tested. As the variance of the estimate depends on the true signal level, a performance measure can be obtained by integrating $V(\hat{M})$ over the required range of signal values, $\left(m_{a}, m_{b}\right)$, for the application being considered. A general form for a performance measure would be

$$
\mathrm{PM}=\int_{m_{a}}^{m_{b}} \frac{h(m)}{\left[g^{\prime}(m)\right]^{2}} d m
$$

The above development of performance measures for measurement systems was based on the assumption that the location function $g(M)$ can be estimated with sufficient precision that it is reasonable to treat it as being known. For many practical situations this is not true and therefore the validity of the developed measures may be questioned. An alternative and more rigorous justification for the SN ratio in (2) is based on the length of Fieller intervals for the true value of $M$. Consider a linear calibration system described by the model

$$
Y=\alpha+\beta M+\sigma \varepsilon, \quad \varepsilon \sim N(0,1) .
$$

Let $y_{j}$ represent the measured values of $Y$, and let $m_{j}$ represent the known values of $M$ for the standards $(j=1, \ldots, p)$. The classical estimates for $\alpha, \beta$ and $\sigma^{2}$ are $\hat{\beta}=S_{y m} / S_{m m}, \hat{\alpha}=\bar{y}-\hat{\beta} \bar{m}$ and $s^{2}=$ $(p-2)^{-1}\left(S_{y y}-\hat{\beta} S_{y m}\right)$, where $S_{y y}=\sum_{1}^{p}\left(y_{j}-\bar{y}\right)^{2}$, $S_{m m}=\sum_{1}^{p}\left(m_{j}-\bar{m}\right)^{2}$, and $S_{y m}=\sum_{1}^{p}\left(y_{j}-\bar{y}\right)\left(m_{j}-\right.$ $\bar{m})$. For a specific value of $M=m_{0}$, the $100(1-\gamma) \%$ prediction interval for $Y$ is given by

$$
\hat{\alpha}+\hat{\beta} m_{0} \pm t s \sqrt{1+\frac{1}{p}+\frac{\left(m_{0}-\bar{m}\right)^{2}}{S_{m m}}},
$$

where $t=t_{\gamma / 2, p-2}$. Suppose that $y_{0}$ is the measured value of $Y$ for a sample which has an unknown value of $M$. A $100(1-\gamma) \%$ confidence interval, called a Fieller interval, for $m_{0}$ can be obtained by using the set of values of $M$ for which $y_{0}$ is in the $100(1-\gamma) \%$ prediction interval of $m$. Therefore, the interval will contain all values of $m$ that satisfy

$$
\left(y_{0}-\hat{\alpha}-\hat{\beta} m\right)^{2} \leq t^{2} s^{2}\left(1+\frac{1}{p}+\frac{(m-\bar{m})^{2}}{S_{m m}}\right) \text {. }
$$

These values can form (i) a finite interval, (ii) a semi-infinite interval, (iii) two semiinfinite intervals or (iv) the entire real line. Cases (ii), (iii) and (iv) would imply no clear evidence of a relationship between $Y$ and $M$ (see Miller and Wu, 1991, for details). So only case (i) is of practical interest. It is shown in Miller and Wu (1991) that for a finite interval, $\left(m_{L}, m_{U}\right)$, its length is

$$
\begin{aligned}
m_{U}-m_{L}=2 t[ & \left(1+\frac{1}{p}\right)\left(\hat{\omega}-\frac{t^{2}}{S_{m m}}\right) \\
& \left.+\hat{\omega} \frac{\left(m_{o}-\bar{m}\right)^{2}}{S_{m m}}\right]^{1 / 2}\left(\hat{\omega}-\frac{t^{2}}{S_{m m}}\right)^{-1}
\end{aligned}
$$

which depends on $\hat{\omega}=\hat{\beta}^{2} / s^{2}, S_{m m}$ and $m_{0}-\bar{m}$. This is equivalent to a result shown by Hoadley (1970) that the width of the Fieller interval depends on the magnitude of the $F$-statistic, $f=S_{m m} \hat{\omega}$, for testing $H: \beta=0$.

The length of the Fieller interval decreases as $\hat{\omega}$ increases for $\hat{\omega}>t^{2} / S_{m m}$. This can easily be seen 
by rewriting (8) as

$$
\begin{aligned}
m_{U}- & m_{L} \\
= & 2 t \\
& {\left[1+\frac{1}{p}+\hat{\omega}\left(\hat{\omega}-\frac{t^{2}}{S_{m m}}\right)^{-1} \frac{\left(m_{0}-\bar{m}\right)^{2}}{S_{m m}}\right]^{1 / 2} } \\
& \cdot\left(\hat{\omega}-\frac{t^{2}}{S_{m m}}\right)^{-1 / 2} .
\end{aligned}
$$

The result is evident since both $\hat{\omega}\left(\hat{\omega}-t^{2} / S_{m m}\right)^{-1}$ and $\left(\hat{\omega}-t^{2} / S_{m m}\right)^{-1 / 2}$ are decreasing functions for $\hat{\omega}>t^{2} / S_{m m}$.

Since the observed length of the Fieller interval is a random variable, the goal of the experiment can be thought of as making the distribution of this variable as favorable as possible. Noting that $S_{m m} \omega$ has a noncentral $F$-distribution with 1 and $\nu=p-2$ degrees of freedom, $E(\hat{\omega})=\nu(\nu-2)^{-1}\left(S_{m m}^{-1}+\omega\right)$, which means that the expected length of the Fieller interval will decrease as $\omega$ decreases. This justifies the maximization of the dynamic SN ratio, $\log \hat{\omega}$, described in (2).

\subsection{Multiple Target Systems}

For multiple target systems the signal factor is used to adjust the function of the system to accommodate different target values for the response. The shape of the signal-response function is not of direct concern for these applications as long as all the desired target values can be realized. Let $\mathscr{M}=\left(m_{a}, m_{b}\right)$ represent the useful range of signal which in practice can be applied to the system, and let $\mathscr{T}$ represent the required target values; $\mathscr{T}$ may either be a set of discrete values or an interval. In this section only results for discrete $\mathscr{T}$ will be presented as the extensions to continuous $\mathscr{T}$ are straightforward.

An obvious way to obtain a performance measure in this situation is to take an average (possibly weighted) of performance for the individual elements of $\mathscr{T}$. Assume that $V(Y)$ given $E(Y)=y_{t}$ is a suitable performance measure, as would be the case if a quadratic loss function is applicable. Then a suitable performance measure can be defined by

$$
\mathrm{PM}=\sum_{y_{t} \in \mathscr{T}} \mathrm{V}\left(Y \mid M=m_{t}\right) w\left(y_{t}\right)
$$

for discrete $\mathscr{T}$, where $m_{t}=g^{-1}\left(y_{t}\right)$ and $w\left(y_{t}\right)$ is a weighting function based on the relative importance of the various targets. In some cases it may not be possible to set $M$ so that $E(Y)=y_{t}$ for all $y_{t}$ in $\mathscr{T}$. In these cases, the PM can be modified by replacing $V(Y)$ by the minimum obtainable mean square error.
The PM in (10) is suitable for multiple target systems which are used for a single purpose. Occasionally, one may encounter a multipurpose system. For example, an injection molding machine may be used to inject a number of different molding materials. As the physical properties of these materials may be quite diverse, the signal-response relationship may vary substantially with respect to the materials. One approach would be to identify compromise settings of the control factors such that the system performs reasonably well over the range of materials. In this case, a weighted average of the performance for individual materials could be used as an overall performance measure. Alternatively, the control factor settings could be optimized for each material individually. This approach may not be practical for control factors which are very difficult to set. In practice, compromise settings can be obtained for those control factors which are difficult to set, and customized settings can be obtained for the rest.

In view of Taguchi's recommendation of the dynamic SN ratio (2) and its prevalent use in some industrial sectors, it is important to point out why it is not appropriate for multiple target systems. Roughly speaking, maximizing $\log \hat{\omega}$ has the effect of minimizing $s^{2}$ and maximizing $\hat{\beta}^{2}$. The former is always desirable, while the latter can lead to undesirable results. For fixed $M=\left(m_{a}, m_{b}\right)$, a larger $|\hat{\beta}|$ value can give a wider range of the $Y$ values, which may be outside the specification limits of the target. Furthermore, if there is error in the setting of $M$ (there is some indication of this in the injection molding example), this error will be propagated through a larger slope $|\hat{\beta}|$, again resulting in a bigger variation in $Y$.

\section{MODELING AND ANALYSIS}

The purpose of a RPD experiment is to identify the manner in which control factors affect the performance of the system. Therefore, the goal is to model the chosen PM as a function of the control factors. However, there are two distinct approaches to developing such a model, which we refer to as performance measure modeling (PMM) and response function modeling (RFM).

Performance measure modeling (PMM) requires a two-stage modeling procedure. The first stage is to obtain an estimate of the PM for each combination of control factors used in the experiment. For a fixed combination of control factor levels, the response is measured for various combinations of signal and noise factor levels and these observations are used to estimate the PM. The second stage in- 
volves using these estimates to model the PM as a function of the control factors. The preferred settings of the control factors are determined directly from this fitted model.

Performance measure modeling can be illustrated using Taguchi's dynamic SN ratio approach. Consider a full factorial experiment involving three twolevel control factors $\left(C_{1}, C_{2}, C_{3}\right)$, two two-level noise factors $\left(N_{1}, N_{2}\right)$ and a four-level signal factor $(M)$. For each of the 8 distinct control factor combinations there are 16 observations corresponding to the noise and signal factor combinations. These 16 observations are used to fit a linear model, $E(Y)=\beta M$, and the parameter estimates are then used to estimate the $\mathrm{PM}$, in this case $\widehat{\mathrm{PM}}=\hat{\beta}^{2} / s^{2}$. Now the eight $\widehat{\mathrm{PM}} \mathrm{s}$ are treated as the set of observations for a $2^{3}$ experiment where $C_{1}, C_{2}$ and $C_{3}$ are the experimental factors and standard analysis techniques can be applied to them. A typical analysis would be to use a normal plot to identify active effects and thus identify a suitable model for PM as a function of the control factors.

Response function modeling (RFM) uses the experimental data to model the signal-response relationship as a function of the control and noise factors. The specified performance measure is then evaluated with respect to the fitted models in order to select preferred levels of the control factors. This approach in essence treats the signal-response relationship as the response and models this relationship as a function of the control and noise factors. This is an extension of the response modeling approach recommended by Welch, Yu, Kang and Sacks (1990) and Shoemaker, Tsui and Wu (1991) for simple response applications.

To illustrate the RFM approach, consider the previous example. The experiment contains 32 combinations of control and noise factor levels. For each of these combinations there are four observations corresponding to the levels of the signal factor. These four observations are used to fit a parametric model for the signal-response relationship. Suppose in our case a model of the form

$$
Y=\beta M+\varepsilon, \quad \varepsilon \sim N\left(0, \sigma^{2}\right)
$$

is suitable. Then $\beta$ and $\sigma^{2}$ are estimated for each of the $32 \mathrm{control} /$ noise factor combinations, and models for $\beta$ and $\sigma^{2}$ as functions of the control and noise factors are produced. The chosen PM is then evaluated for different combinations of the control factors using these models. A systematic development of RFM is given in Miller and Wu (1991).

We do not, in general, recommend the PMM procedure since it can often obscure useful information present in the data. Box (1988) illustrates for sim- ple response systems how very different data sets can give the same estimated values of performance measures. Therefore, modeling the PM directly can result in the loss of valuable information regarding the problem. Clearly, the same argument applies to signal-response applications. The PPM approach only provides information on how control factors affect the overall performance of the system. Any information in the data on how specific control factors affect the shape of the signal-response system or interact with specific noise factors is lost. It is this type of information which can be most valuable in suggesting directions for future research. The response-based approaches do not suffer from this deficiency. The initial modeling of the response will often provide useful insight into the system and may suggest directions for future research. The PM is then applied to this model to identify preferred settings of control factors. In many cases, it will not be necessary to do this step formally since it will be straightforward to deduce the preferred settings directly from the response model. The advantages of the RFM approach will be clearly demonstrated in Section 5 using the injection molding experiment.

\section{ANALYSIS OF THE INJECTION MOLDING EXPERIMENT}

In this section, data from the injection molding experiment is used to demonstrate the PMM and RFM approaches. The Appendix contains the data from the original experiment (DeMates, 1990).

First, a suitable PM must be identified. Suppose it is required that the system is capable of achieving target values from 650 to 700 . Since this range of target values is obtainable for all combinations of control and noise factors used in the experiment, a reasonable PM would be the variation of the response over this range.

The experiment was run over two days using a split-plot randomization procedure. On the first day, the compound noise factor was set to its low level and the control factors were then varied using a $2^{7-4}$ factorial design (see Table 3). For each control factor combination, the signal factor was varied over its eight levels and four observations were taken at each level. On the second day, the procedure was repeated using the high level of the compound noise factor.

Figure 2 contains scatter plots of the data $(Y, M)$ and the fitted quadratic models for several runs, where the response $Y$ is the part weight and the signal factor $M$ is the high injection pressure (see Section 2). Figure 3 contains the residual plots (after the quadratic model fit) against $M$. Although 
TABLE 3

Primary array for injection molding case study

Control Factors

\begin{tabular}{crrrrrrr}
\cline { 2 - 7 } Row & A & B & C & D & E & F & G \\
\hline 1 & 1 & 1 & 1 & 1 & 1 & 1 & 1 \\
2 & 1 & 1 & 1 & -1 & -1 & -1 & -1 \\
3 & 1 & -1 & -1 & 1 & 1 & -1 & -1 \\
4 & 1 & -1 & -1 & -1 & -1 & 1 & 1 \\
5 & -1 & 1 & -1 & 1 & -1 & 1 & -1 \\
6 & -1 & 1 & -1 & -1 & 1 & -1 & 1 \\
7 & -1 & -1 & 1 & 1 & -1 & -1 & 1 \\
8 & -1 & -1 & 1 & -1 & 1 & 1 & -1 \\
\hline
\end{tabular}

the quadratic model appears reasonably satisfactory, there appears to be a systematic pattern in the residual plots. In order to investigate this further, the total sum of squares for each control/noise factor combination was divided into contrasts using orthogonal polynomials of $M$. Then a forward selection procedure was used to test sequentially the addition of higher-order terms (at the 0.05 significance level) to the models.

In 12 of the 16 cases, the selection procedure indicated that a quadratic polynomial was adequate. In the other four cases, the cubic term was also added. An interesting aspect of this analysis was that in over half the runs there was an unusually large sum of squares attributed to the sixth degree term (it was typically more than a factor of 10 larger than the fourth or fifth degree terms). As it is unlikely that the sixth degree term is important this suggests that at least part of the systematic pattern in the residuals (after quadratic fit) seen in Figure 3 may not be due to model inadequacy. One possible explanation is that a systematic difference exists between the settings of the signal factor and

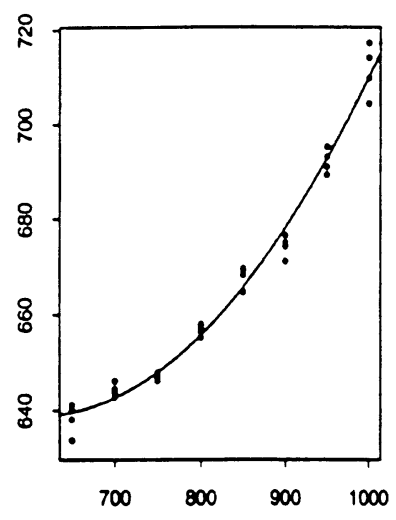

$X_{N}=1$ Row 1

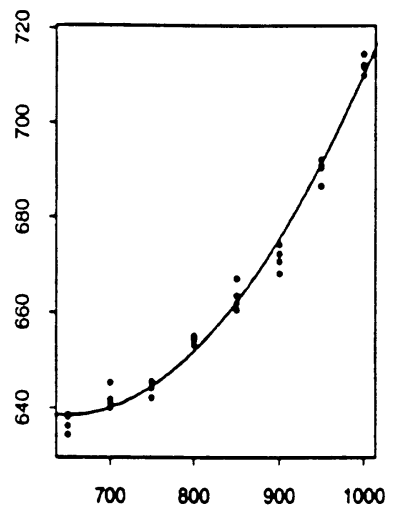

$X_{N}=1$ Row 2
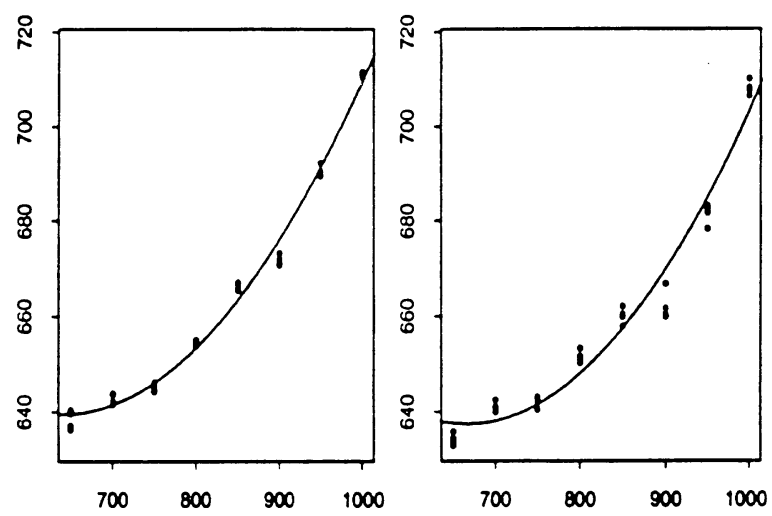

$X_{N}=-1$ Row 1

$X_{N}=-1$ Row 2

FIG. 2. Fitted quadratic models for injection molding data: (a) $X_{N}=1$, Row 1; (b) $X_{N}=1$, Row 2 ; (c) $X_{N}=-1$, Row 1 ; (d) $X_{N}=-1$, Row 2.
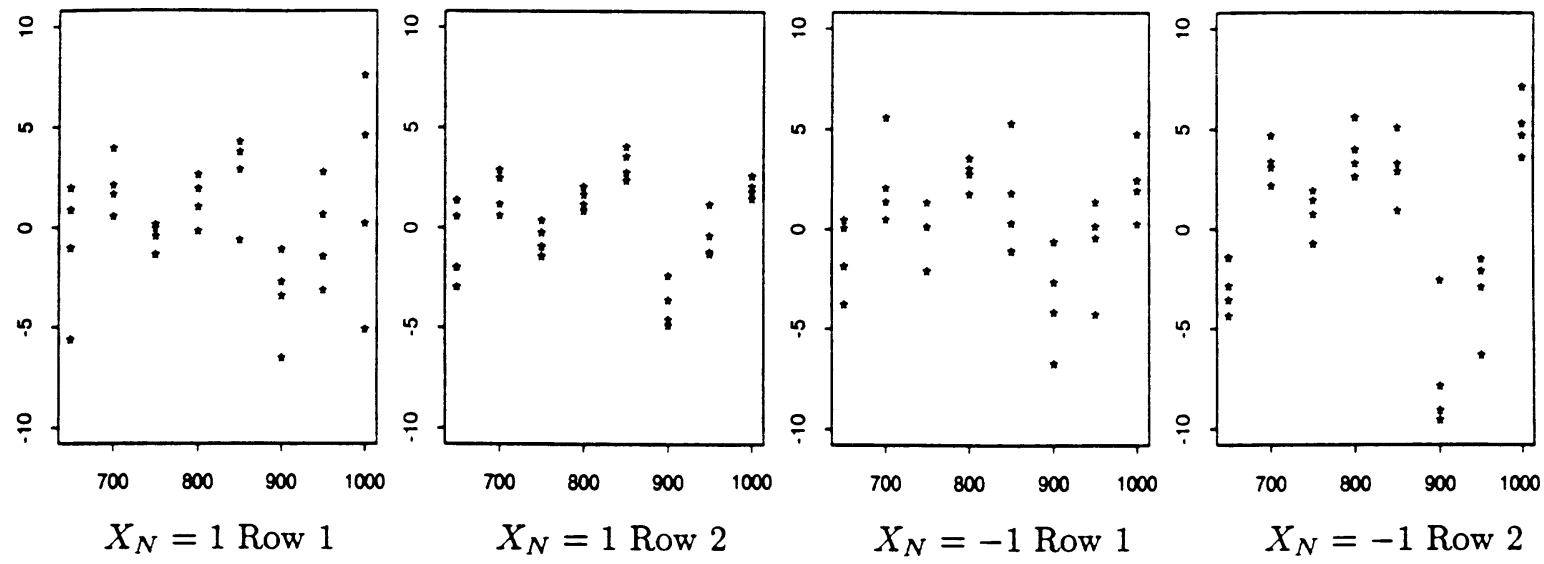

FIG. 3. Residual plots for injection molding data: (a) $X_{N}=1$, Row 1 ; (b) $X_{N}=1$, Row 2; (c) $X_{N}=-1$, Row 1 ; (d) $X_{N}=-1$, Row 2 . 
the actual high injection pressure delivered by the system. For example, the residuals corresponding to $M=900$ are negative in most of the residual plots. This could be explained if the system consistently delivered a high injection pressure of less than 900 at a nominal setting of 900 , since this would cause the observed part weights to be lower than expected. Whatever the real explanation for this phenomenon, it is clear it will not be corrected by using a more complicated model.

Figure 2 indicates that the quadratic model adequately captures the essential features of the signal-response relationship. Although for certain runs the above analysis indicated that the cubic term was significant, from a practical point of view it does not appear necessary. Therefore, a quadratic location model based on orthogonal polynomials was used,

$$
E(Y)=\beta_{0}+\beta_{1} P_{1}(M)+\beta_{2} P_{2}(M),
$$

where the values $P_{1}(M)$ and $P_{2}(M)$ for the various signal levels are given in Table 4 . This type of model was adopted as it makes the interpretation of results easier with $\beta_{1}$ representing the linear component of the signal-response relationship and $\beta_{2}$ the quadratic departure from linearity.

\subsection{PMM Analysis}

The first step is to estimate the PM for each combination of control factors. From the residual plots it seems reasonable to assume variation is constant across levels of the signal factor. For each row in the control factor array, the standard least squares estimate of variance, $s^{2}$, for the fitted quadratic model (11) was used as the estimated PM. The next step is to treat $s^{2}$ as the response for the control factor design array. Actually, $\log \left(s^{2}\right)$ will be used in order to stabilize the variation of these estimates. Figure 4 gives the half-normal plot of factor effects for $\log \left(s^{2}\right)$. This plot does not clearly indicate that any control factors have an effect on the PM. However, it does appear that A, B, and D warrant further consideration.

\subsection{RFM Analysis}

For RFM, we first fit location and dispersion models for each combination of control and noise factors used in the experiment. For the location model, we use the quadratic model in (11). Since replicate observations were made at each signal level, it is possible to separate variation into a lack-of-fit component and a replicate component. The replicate component $\hat{\sigma}_{p}^{2}$ will reflect part-to-part variation for observations taken over a short time interval. The lack-of-fit component $\hat{\sigma}_{l}^{2}$ will represent
TABLE 4

Levels for orthogonal polynomials

\begin{tabular}{rrrrrrrrc}
\hline & \multicolumn{7}{c}{ Signal factor levels } \\
\cline { 2 - 9 } & 650 & 700 & 750 & 800 & 850 & 900 & 950 & 1,000 \\
\hline$P_{1}(M)$ & -7 & -5 & -3 & -1 & 1 & 3 & 5 & 7 \\
$P_{2}(M)$ & 7 & 1 & -3 & -5 & -5 & -3 & 1 & 7 \\
\hline
\end{tabular}

longer-term variation, and as was noted previously, contains a systematic component which may be due to systematic errors in the recorded values for the signal factor. Therefore, we will analyze these two components of variation separately. The estimated values for each of the location parameters and the two components of variation are given in Table 6 .

Next, the effects of the control and noise factors on these parameters are evaluated. Figure 5 contains half-normal plots for the location model parameters, and Figure 6 contains half-normal plots for the two components of variation. For $\beta_{0}$ there are six effects which appear to be significant: G, C, E, A, N and F. Only $\mathrm{C}$ stands out as being clearly significant for $\beta_{1}$. There are no clearly significant effects for $\beta_{2}$, but E, $\mathrm{N}, \mathrm{D}, \mathrm{B}, \mathrm{AN}, \mathrm{FN}$ and GN appear marginal. None of the estimated effects are significant for lack-of-fit variation, while for the part-to-part variation $\mathrm{A}$ is clearly significant and N, EN, B and C are all large enough to warrant further attention.

The fitted models for the parameters are

$$
\begin{aligned}
\hat{\beta}_{0}= & 666.4+1.2 X_{A}-1.8 X_{C}+1.4 X_{E} \\
& -1.0 X_{F}+1.8 X_{G}+1.1 X_{N}, \\
\hat{\beta}_{1}= & 4.79+0.16 X_{C}, \\
\hat{\beta}_{2}= & 1.33+0.03 X_{B}-0.04 X_{D}-0.05 X_{E} \\
& -0.04 X_{N}+0.01 X_{A} X_{N}-0.03 X_{F} X_{N} \\
& -0.02 X_{G} X_{N} \\
\hat{\nu}= & 0.12+1.10 X_{A}+0.22 X_{B}-0.21 X_{C}, \\
& +0.40 X_{N}+0.28 X_{E} X_{N}+0.04 X_{E},
\end{aligned}
$$

where $\nu=\log \sigma_{p}^{2}$.

TABLE 5

Estimated parameters for PMM

\begin{tabular}{ccccr}
\hline Row & $\hat{\beta_{0}}$ & $\hat{\beta_{1}}$ & $\hat{\beta}_{2}$ & \multicolumn{1}{c}{$s^{2}$} \\
\hline 1 & 665.8 & 5.00 & 1.25 & 8.39 \\
2 & 662.2 & 4.91 & 1.46 & 19.70 \\
3 & 666.8 & 4.93 & 1.25 & 9.06 \\
4 & 666.4 & 4.66 & 1.40 & 10.40 \\
5 & 665.3 & 4.56 & 1.38 & 4.34 \\
6 & 674.3 & 4.33 & 1.34 & 9.75 \\
7 & 666.4 & 4.92 & 1.31 & 1.54 \\
8 & 664.3 & 4.96 & 1.27 & 3.27 \\
\hline
\end{tabular}




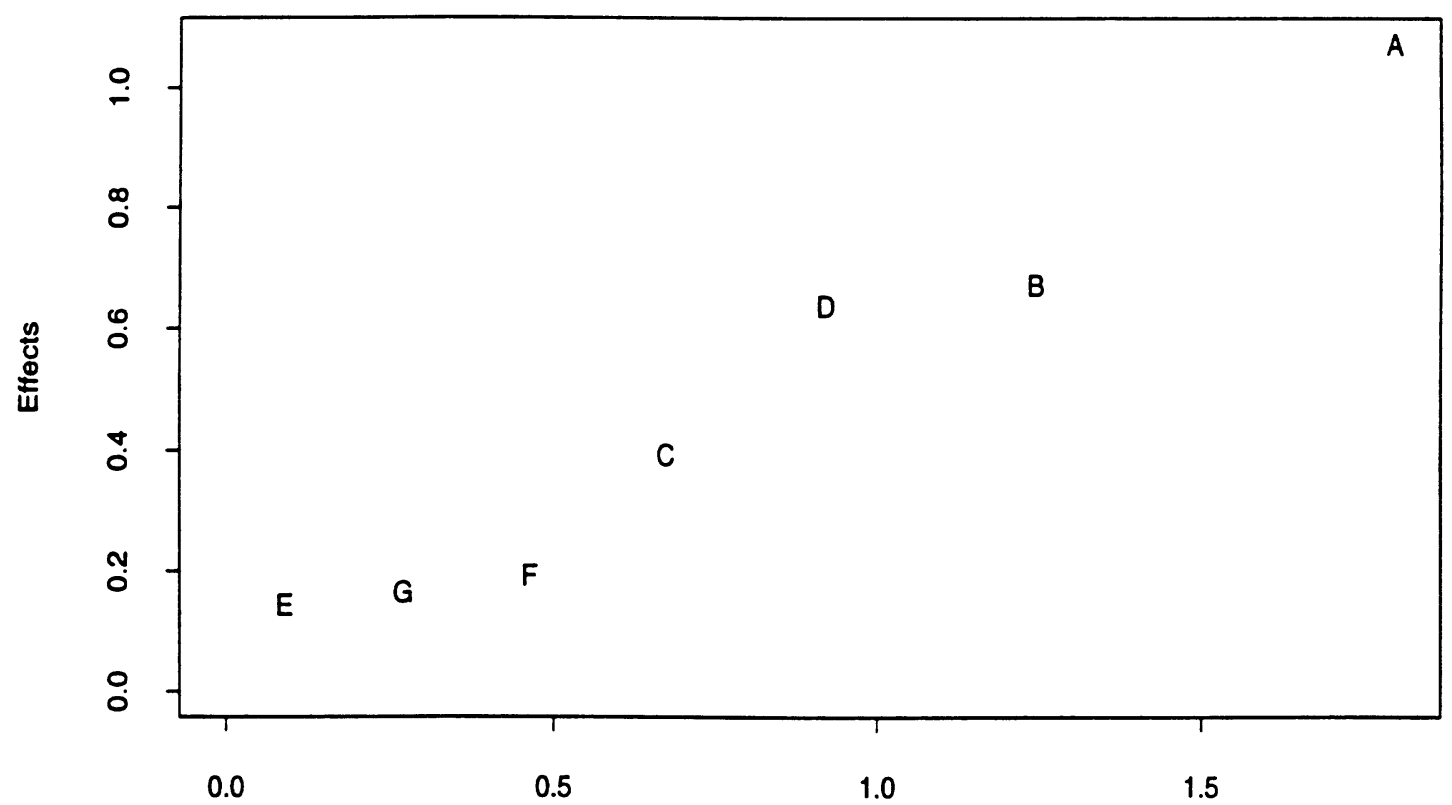

FIG. 4. Half-normal Plot for $\log \left(s^{2}\right)$.

TABLE 6

Estimated parameters for injection molding data

\begin{tabular}{|c|c|c|c|c|c|c|c|c|c|c|c|}
\hline \multicolumn{6}{|c|}{$X_{N}=1$} & \multicolumn{6}{|c|}{$X_{N}=-1$} \\
\hline Run & $\hat{\beta_{0}}$ & $\hat{\beta_{1}}$ & $\hat{\beta_{2}}$ & $\hat{\sigma_{l}^{2}}$ & $\hat{\sigma_{p}^{2}}$ & Run & $\hat{\beta_{0}}$ & $\hat{\beta_{1}}$ & $\hat{\beta_{2}}$ & $\hat{\sigma_{l}^{2}}$ & $\hat{\sigma_{p}^{2}}$ \\
\hline 1 & 666.5 & 5.02 & 1.16 & 5.61 & 7.78 & 1 & 665.0 & 4.98 & 1.33 & 6.87 & 1.20 \\
\hline 2 & 664.2 & 5.12 & 1.44 & 7.10 & 4.45 & 2 & 660.0 & 4.69 & 1.48 & 26.81 & 3.20 \\
\hline 3 & 668.2 & 4.98 & 1.22 & 4.28 & 4.99 & 3 & 665.2 & 4.86 & 1.26 & 6.34 & 2.70 \\
\hline 4 & 668.4 & 4.76 & 1.25 & 4.81 & 3.53 & 4 & 664.2 & 4.55 & 1.54 & 3.64 & 2.64 \\
\hline 5 & 666.3 & 4.66 & 1.35 & 4.93 & 0.67 & 5 & 664.2 & 4.46 & 1.39 & 2.54 & 0.56 \\
\hline 6 & 674.4 & 4.32 & 1.32 & 14.78 & 1.00 & 6 & 674.1 & 4.33 & 1.36 & 13.27 & 0.30 \\
\hline 7 & 666.6 & 4.92 & 1.31 & 2.30 & 0.21 & 7 & 666.1 & 4.91 & 1.30 & 1.76 & 0.18 \\
\hline 8 & 664.9 & 4.90 & 1.25 & 3.21 & 0.75 & 8 & 663.6 & 5.02 & 1.29 & 3.96 & 0.12 \\
\hline
\end{tabular}

To begin, consider the fitted model for $\beta_{1}$. Factor $\mathrm{C}$ can be used to adjust the sensitivity of the response to the signal. In this case $\mathrm{C}$ would be set to level $X_{C}=1$ if a wider range of attainable targets was necessary. Otherwise the level of $\mathrm{C}$ could be determined by other considerations.

Next consider the model for $\log \sigma_{p}^{2}$. Part-to-part variation is not the only type of variation which is relevant to the process. However, it is clearly desirable to reduce this type of variation as much as possible. In this case, A should be set to the $X_{A}=-1$ level. It also appears worthwhile to set $B$ to the $X_{B}=-1$ level and $\mathrm{C}$ to the $X_{C}=1$ level. The compound noise factor $\mathrm{N}$ and the $\mathrm{EN}$ interaction also affect $\sigma_{p}^{2}$. Since the EN interaction was significant, $\mathrm{E}$ was also included in the model. Table 7 contains the estimated values of $\log \sigma_{p}^{2}$ for the combinations of levels of $\mathrm{E}$ and $\mathrm{N}$ assuming $X_{A}=-1, X_{B}=-1$ and $X_{C}=1$. The table indicates that for $\mathrm{E}$ set to
$X_{E}=-1$ the part-to-part variation will be more consistent with respect to changes in the noise factors than for $X_{E}=1$.

Now consider the model for $\beta_{0}$. Due to previous considerations the levels of $\mathrm{A}, \mathrm{C}$ and $\mathrm{E}$ have already been determined. This leaves factors $\mathrm{F}$ and $\mathrm{G}$ which could be used to make adjustments to $\beta_{0}$ (if necessary). Notice, that $\mathrm{N}$ does affect $\beta_{0}$ but no interaction has been identified which could offset this.

Finally, consider the model for $\beta_{2}$. In this case the levels for $\mathrm{B}, \mathrm{D}$ and $\mathrm{E}$ have been previously determined. Consider the effect of $\mathrm{N}$. The estimated observed coefficient (given fixed levels of control factors) for $\mathrm{N}$ would be

$$
\left(-0.04+0.01 X_{A}-0.03 X_{F}-0.02 X_{G}\right) X_{N} .
$$

In order to make the system insensitive to changes in $\mathrm{N}$, we would like to make the absolute value of 

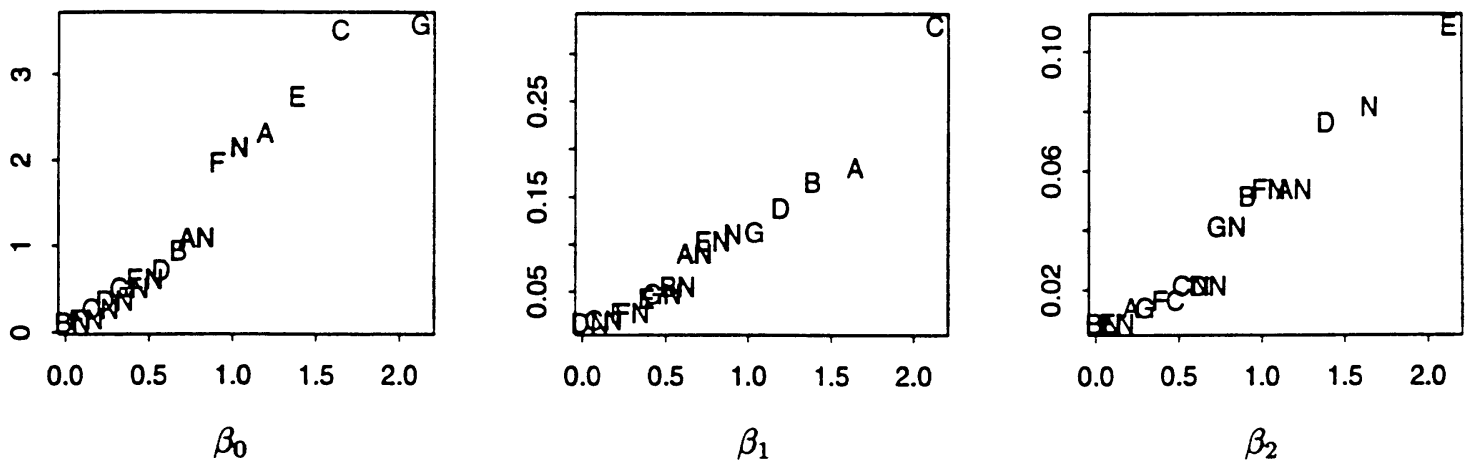

FIG. 5. Half-normal plots for location model parameters: (a) $\beta_{0}$; (b) $\beta_{1}$; (c) $\beta_{2}$.
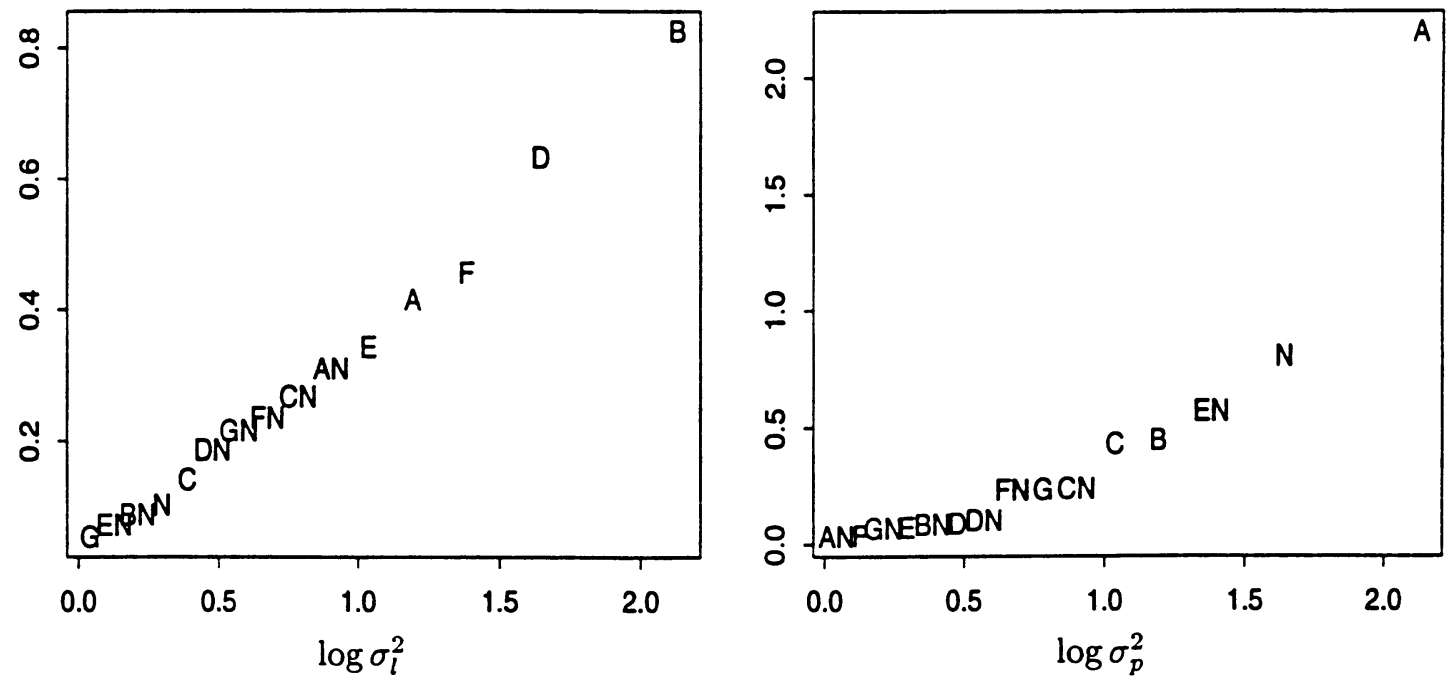

FIG. 6. Half-normal plot for variance parameters: (a) $\log \sigma_{l}^{2}$; (b) $\log \sigma_{p}^{2}$.

this coefficient as small as possible. As the setting $X_{A}=-1$ has already been determined, this suggests setting $X_{F}=-1$ and $X_{G}=-1$.

Notice that the PMM approach did not clearly indicate that any control factors could be adjusted to improve system performance. Even if certain factors had been identified, it would not have provided insight into how these factors affect the system. On the other hand, RFM not only indicated certain control factors could be used to improve the system, it also provided insight into how these factors affected the system. In particular a quadratic model was

TABLE 7

Estimated Values for $\log \sigma_{p}^{2}$

\begin{tabular}{lcc}
\hline & $X_{E}=-1$ & $X_{E}=1$ \\
\hline$X_{N}=-1$ & -1.57 & -2.05 \\
$X_{N}=1$ & -1.33 & -0.69 \\
\hline
\end{tabular}

identified as suitably describing the signalresponse relationship and control factors which could be used to alter the parameters of this relationship were identified. Further, the residual plots produced from the fitted models for the signalresponse relationship indicated the possibility of a systematic error in the signal levels. Finally, the flexibility of the RFM procedure allowed variation to be divided into two components, $\sigma_{p}^{2}$ and $\sigma_{l}^{2}$, which led to the conclusion that factor A could be used to reduce part-to-part variability.

The advantages of the RFM approach can be better appreciated by contrasting the main findings summarized above with those obtained using Taguchi's approach. First, Taguchi's dynamic SN ratio assumes a linear relation while the data clearly exhibit a quadratic relation. Second, use of data analysis techniques, like residual plots suggests the possibility of errors in the signal factor setting. Third, analyzing components of variation 
allows us to identify factor A as effective in reducing part-to-part variability, which can have major engineering implications.

\section{DESIGNING EXPERIMENTS}

The experiment used for the injection molding experiment is typical of many RPD experiments which use a PMM approach to analysis. High injection pressure was used as the signal factor since it was known to have a strong influence on the response (part weight). Four key causes of variability in part weight were identified: melt index, percent regrind; operator; and resin moisture. A single compound noise factor was defined to represent levels of these factors (see Table 2). Seven control factors were identified (see Table 1). These represented settings of the injection molding apparatus that were thought to have the potential of influencing the signal-response relationship.

The original experiment was designed on the basis that PMM was to be used to analyze the data. This requires the PM to be estimated for each combination of control factors used in the experiment. It is prudent to use the same combinations of noise and signal factors to obtain each estimate, since the choice of combinations can affect the estimate. Therefore the design array should be constructed as the "product" of a primary array which is used to vary levels of the control factors and a secondary array which is used to vary levels of the noise and signal factors. The overall design array consists of replicating the secondary array, for each row in the primary array which Shoemaker, Tsui and $\mathrm{Wu}$ (1991) referred to as a product array design. The total number of runs will be the number of rows in the primary array times the number of rows in the secondary array. Often fractional factorial designs are used for one or both of these arrays to reduce the size of the experiment.

For the injection molding experiment a $2^{7-4}$ design was used for the primary array (see Table 3). This allows seven control factors to be investigated using just eight runs. However, all control factor interactions are confounded with control factor main effects, and the results may be misleading if any of these interactions are nonnegligible. For each of the 8 rows of the primary array 16 observations were taken corresponding to all combinations of levels for the compound noise factor (2 levels) and the signal factor ( 8 levels). The compound noise factor is, in essence, four noise factors which have been completely confounded with each other. This approach is risky and should only be used if the manner in which the noise factors affect the system is known to satisfy certain conditions. The combinations of levels used to define the levels of the compound noise factor should represent the extreme sets of conditions for all settings of the control factors. For example, assume that the individual conditions assigned to $X_{N}=-1$ are known always to decrease part weight and those assigned to $X_{N}=+1$ always to increase part weight. Further, for simplicity, assume the effects of the individual noise factors are additive so that $X_{N}=+1$ and $X_{N}=-1$ represent the extreme cases. Under these circumstances, a reduction in the difference in part weights between $X_{N}=+1$ and $X_{N}=-1$ can reasonably be expected to result in an overall reduction of variation in part weight. On the other hand, consider the consequences if the new operator tends to produce parts which are lighter than those produced by the experienced operator for some control factor settings but produces heavier parts for other settings. For the second group of control factor settings, the effect of the operator will tend to offset the effects of the other noise factors which will reduce the difference in part weights between $X_{N}=+1$ and $X_{N}=-1$. However, for these control factor settings $X_{N}=+1$ and $X_{N}=-1$ do not represent the extreme cases (the extreme cases would have the reverse levels of operator) and so the variation in part weights is not necessarily reduced at these settings. A second consequence of using a compound noise factor is that the effects of the individual noise factors cannot be separated. This limits the usefulness of the design in terms of the information it provides about directions for future research. If, for example, setting factor A (injection speed) to the +1 level had been found to improve the PM, there is no way of determining the nature of the improvement. Is the process more robust to operators, percent regrind, melt index, resin moisture or some combination of these? Answers to questions like this will indicate which sources of variability can be addressed using factor $\mathrm{A}$ and which sources require other solutions.

One main difficulty associated with using a product array design with the noise factors in a different array from the control factors (as is required for a PMM approach) is that it can lead to inefficient designs. Consider the injection molding experiment and assume that cost is mainly determined by the number of control/noise factor combinations which are used (this is a reasonable assumption for many signal-response system experiments, as the signal factor is usually relatively easy to adjust in comparison to control/noise factors). The actual experiment only used a total of 16 control/noise factor combinations, but to achieve this it was necessary to use a resolution III design for the primary array ( 7 fac- 
TABLE 8

Comparison of resolution of designs

\begin{tabular}{ccc}
\hline $\begin{array}{c}\text { Noise-control } \\
\text { combinations }\end{array}$ & $\begin{array}{c}\text { Product array } \\
\text { for PMM }\end{array}$ & $\begin{array}{c}\text { Product array } \\
\text { for RFM }\end{array}$ \\
\hline 16 & II & III \\
32 & II & IV \\
64 & III & IV \\
128 & IV & V \\
\hline
\end{tabular}

tors) and what was effectively a resolution II design for the secondary array (4 factors). Suppose a design was wanted which used resolution IV designs for both the primary and secondary arrays. This would mean that the primary array must contain at least 16 rows and the secondary array must contain at least 8 rows resulting in a minimum of 128 control/noise combinations (see Box, Hunter and Hunter, 1978, page 410) for a table of maximum resolution designs). Contrast this to the situation when an RFM approach is used. For RFM a fitted model of the signal-response relationship is obtained for each combination of control and noise factors used in the experiment, which means the same set of signal levels should be used for each combination of control/noise factors. A product array design should be used, but in this case the primary array contains levels of both the control and noise factors and the secondary array only contains levels for the signal factor. For the injection molding experiment, one can construct a 32-run primary array for the 7 control factors and 4 noise factors which has resolution IV (Box, Hunter and Hunter, 1978, page 410). Table 8 compares the maximum resolution of designs which can be constructed using each of the product array approaches for given numbers of control/noise combinations. Table 9 contains an example of a 16-combination, resolution III array which is suitable for RFM and could have been used instead of the design described in Section 5. Notice this array utilizes all 16 possible combinations of the 4 noise factors compared to the 2 combinations which were actually used.

The above discussion focused on reducing the number of control/noise combinations. However, in some situations certain control/noise factors are more difficult or expensive to set than others. In these cases, cost is determined by the number of level changes of the "difficult-to-change" factors and designs which involve restricted randomization such as split-plot or strip-plot designs may be useful in reducing cost. Difficult-to-change factors are assigned to main-units which reduces the number of times these factors are reset. Further discussion can be found in Box and Jones (1992). In considering fractional factorial design matrices which are suitable for split-plot designs, the key characteristic is the number of distinct combinations of the difficult-to-change factors in the design matrix. This number can be reduced by having as many generators as possible in the defining relation made entirely from the difficult-to-change factors. Consider combining this requirement with the restrictions imposed by the modeling procedures. For RFM all control and noise factors are included in the primary array and therefore in selecting the primary array as many generators as possible should be made from difficult-to-change factors. For PMM the control and noise factors are split between the primary and secondary arrays and so the difficult-to-change factors may also be split. This can severely restrict the ability to select generators made entirely from difficult-to-change factors.

\section{CONCLUSION}

This paper explored the use of designed experiments to improve the performance of signalresponse systems. These are systems whose function depends on the causal relationship between a signal factor and a response variable. Measurement systems and multiple target systems are examples of such systems and were considered in detail. To summarize we recommend the following steps for investigating signal-response systems.

1. Identify a suitable performance measure which reflects the ability of the system to perform its designated function.

2. Adopt a response function modeling approach. In essence this means that the signal-response relationship is to be modeled as a function of both control and noise factors. Then the identified performance measure is applied to the fitted model to determine preferred settings for the control factors.

3. The experiment should be designed using a twostage strategy. First, a design array is adopted for the control and noise factors. Then, for each row in this array, the signal factor is varied over a number of levels.

4. The analysis is quite straightforward. For each row in the control-noise array, parametric location and dispersion models are fitted for the response. The fitted parameters for these models are then modeled as functions of the control and noise factors. Standard procedures, such as half normal plots and regression analysis, are used to identify significant effects and produce a fitted model. The PM is then applied to this model and preferred settings of the control factors are identified. 
TABLE 9

A 16 run, resolution III design for 7 control factors and 4 noise factors

\begin{tabular}{|c|c|c|c|c|c|c|c|c|c|c|c|}
\hline \multirow[b]{2}{*}{ Run } & \multicolumn{7}{|c|}{ Control factors } & \multicolumn{4}{|c|}{ Noise factors } \\
\hline & $A$ & $B$ & $C$ & $D$ & $E$ & $F$ & $G$ & $N$ & $O$ & $P$ & $Q$ \\
\hline 1 & -1 & -1 & -1 & -1 & +1 & +1 & +1 & -1 & -1 & -1 & +1 \\
\hline 2 & +1 & -1 & -1 & -1 & -1 & -1 & -1 & +1 & +1 & -1 & -1 \\
\hline 3 & -1 & +1 & -1 & -1 & -1 & +1 & +1 & +1 & -1 & +1 & -1 \\
\hline 4 & +1 & +1 & -1 & -1 & +1 & -1 & -1 & -1 & +1 & +1 & +1 \\
\hline 5 & -1 & -1 & +1 & -1 & +1 & -1 & +1 & -1 & +1 & +1 & -1 \\
\hline 6 & +1 & -1 & +1 & -1 & -1 & +1 & -1 & +1 & -1 & +1 & +1 \\
\hline 7 & -1 & +1 & +1 & -1 & -1 & -1 & +1 & +1 & +1 & -1 & +1 \\
\hline 8 & +1 & +1 & +1 & -1 & +1 & +1 & -1 & -1 & -1 & -1 & -1 \\
\hline 9 & -1 & -1 & -1 & +1 & +1 & +1 & -1 & +1 & +1 & +1 & -1 \\
\hline 10 & +1 & -1 & -1 & +1 & -1 & -1 & +1 & -1 & -1 & +1 & +1 \\
\hline 11 & -1 & +1 & -1 & +1 & -1 & +1 & -1 & -1 & +1 & -1 & +1 \\
\hline 12 & +1 & +1 & -1 & +1 & +1 & -1 & +1 & +1 & -1 & -1 & -1 \\
\hline 13 & -1 & -1 & +1 & +1 & +1 & -1 & -1 & +1 & -1 & -1 & +1 \\
\hline 14 & +1 & -1 & +1 & +1 & -1 & +1 & +1 & -1 & +1 & -1 & -1 \\
\hline 15 & -1 & +1 & +1 & +1 & -1 & -1 & -1 & -1 & -1 & +1 & -1 \\
\hline 16 & +1 & +1 & +1 & +1 & +1 & +1 & +1 & +1 & +1 & +1 & +1 \\
\hline
\end{tabular}

APPENDIX: DATA FROM THE INJECTION MOLDING EXPERIMENT

TABLE A1

\begin{tabular}{|c|c|c|c|c|c|c|c|c|c|}
\hline \multirow[b]{2}{*}{ Run } & \multicolumn{8}{|c|}{ Signal factor levels } & \multirow[b]{2}{*}{$X_{N}$} \\
\hline & 650 & 700 & 750 & 800 & 850 & 900 & 950 & 1,000 & \\
\hline 1 & $\begin{array}{l}640.1 \\
641.2 \\
633.6 \\
638.2\end{array}$ & $\begin{array}{l}644.4 \\
646.2 \\
642.8 \\
643.9\end{array}$ & $\begin{array}{l}647.6 \\
646.3 \\
647.2 \\
647.8\end{array}$ & $\begin{array}{l}655.2 \\
657.3 \\
656.4 \\
658.0\end{array}$ & $\begin{array}{l}664.8 \\
669.7 \\
668.3 \\
669.2\end{array}$ & $\begin{array}{l}674.4 \\
671.3 \\
676.7 \\
675.1\end{array}$ & $\begin{array}{l}693.2 \\
689.4 \\
691.1 \\
695.3\end{array}$ & $\begin{array}{l}709.8 \\
714.2 \\
717.2 \\
704.5\end{array}$ & 1 \\
\hline 2 & $\begin{array}{l}638.6 \\
636.3 \\
634.4 \\
638.2\end{array}$ & $\begin{array}{l}645.3 \\
640.2 \\
641.8 \\
641.1\end{array}$ & $\begin{array}{l}645.5 \\
642.1 \\
642.1 \\
644.3\end{array}$ & $\begin{array}{l}655.1 \\
654.3 \\
653.3 \\
654.6\end{array}$ & $\begin{array}{l}662.1 \\
663.6 \\
660.7 \\
667.1\end{array}$ & $\begin{array}{l}670.8 \\
668.2 \\
672.3 \\
674.3\end{array}$ & $\begin{array}{l}692.3 \\
691.1 \\
690.5 \\
686.7\end{array}$ & $\begin{array}{l}711.8 \\
712.3 \\
714.6 \\
710.1\end{array}$ & 1 \\
\hline 3 & $\begin{array}{l}642.6 \\
640.2 \\
641.6 \\
639.9\end{array}$ & $\begin{array}{l}648.3 \\
642.9 \\
646.1 \\
645.2\end{array}$ & $\begin{array}{l}650.0 \\
648.2 \\
647.9 \\
649.9\end{array}$ & $\begin{array}{l}657.3 \\
659.4 \\
658.1 \\
660.0\end{array}$ & $\begin{array}{l}666.3 \\
667.3 \\
670.1 \\
671.5\end{array}$ & $\begin{array}{l}675.2 \\
674.4 \\
676.6 \\
678.2\end{array}$ & $\begin{array}{l}695.2 \\
691.4 \\
689.9 \\
699.2\end{array}$ & $\begin{array}{l}714.6 \\
713.7 \\
714.2 \\
709.9\end{array}$ & 1 \\
\hline 4 & $\begin{array}{l}643.8 \\
641.6 \\
642.2 \\
643.6\end{array}$ & $\begin{array}{l}649.8 \\
646.3 \\
645.2 \\
647.2\end{array}$ & $\begin{array}{l}650.6 \\
649.7 \\
648.2 \\
650.1\end{array}$ & $\begin{array}{l}658.3 \\
657.9 \\
659.1 \\
660.0\end{array}$ & $\begin{array}{l}666.2 \\
666.8 \\
670.2 \\
671.8\end{array}$ & $\begin{array}{l}673.2 \\
675.8 \\
675.8 \\
678.2\end{array}$ & $\begin{array}{l}696.6 \\
691.2 \\
690.2 \\
690.6\end{array}$ & $\begin{array}{l}713.8 \\
711.7 \\
711.8 \\
712.2\end{array}$ & 1 \\
\hline 5 & $\begin{array}{l}642.6 \\
641.8 \\
642.0 \\
642.3\end{array}$ & $\begin{array}{l}645.6 \\
645.8 \\
645.7 \\
646.0\end{array}$ & $\begin{array}{l}647.9 \\
648.2 \\
648.0 \\
647.8\end{array}$ & $\begin{array}{l}654.6 \\
655.2 \\
654.7 \\
654.9\end{array}$ & $\begin{array}{l}666.8 \\
665.7 \\
665.8 \\
669.2\end{array}$ & $\begin{array}{l}672.3 \\
674.6 \\
673.9 \\
675.4\end{array}$ & $\begin{array}{l}687.9 \\
688.8 \\
689.3 \\
688.6\end{array}$ & $\begin{array}{l}709.8 \\
710.2 \\
711.3 \\
710.7\end{array}$ & 1 \\
\hline 6 & $\begin{array}{l}650.6 \\
650.2 \\
651.3 \\
650.1\end{array}$ & $\begin{array}{l}655.7 \\
656.2 \\
655.5 \\
656.0\end{array}$ & $\begin{array}{l}660.2 \\
659.7 \\
659.4 \\
658.9\end{array}$ & $\begin{array}{l}667.8 \\
666.5 \\
666.7 \\
666.6\end{array}$ & $\begin{array}{l}671.1 \\
672.0 \\
671.7 \\
671.4\end{array}$ & $\begin{array}{l}678.9 \\
679.3 \\
679.1 \\
678.6\end{array}$ & $\begin{array}{l}694.7 \\
693.3 \\
696.8 \\
692.1\end{array}$ & $\begin{array}{l}718.4 \\
720.2 \\
716.6 \\
717.0\end{array}$ & 1 \\
\hline 7 & $\begin{array}{l}639.9 \\
640.2 \\
640.3 \\
640.1\end{array}$ & $\begin{array}{l}644.1 \\
644.6 \\
645.0 \\
644.7\end{array}$ & $\begin{array}{l}647.6 \\
648.0 \\
648.2 \\
647.8\end{array}$ & $\begin{array}{l}656.3 \\
656.0 \\
656.4 \\
656.7\end{array}$ & $\begin{array}{l}664.8 \\
665.2 \\
665.1 \\
665.5\end{array}$ & $\begin{array}{l}675.3 \\
674.7 \\
674.9 \\
675.2\end{array}$ & $\begin{array}{l}693.1 \\
692.8 \\
691.9 \\
692.4\end{array}$ & $\begin{array}{l}709.9 \\
711.4 \\
712.2 \\
711.6\end{array}$ & 1 \\
\hline 8 & $\begin{array}{l}637.7 \\
638.1 \\
638.2 \\
638.4\end{array}$ & $\begin{array}{l}642.9 \\
643.4 \\
643.0 \\
642.9\end{array}$ & $\begin{array}{l}647.3 \\
647.3 \\
646.8 \\
647.0\end{array}$ & $\begin{array}{l}651.1 \\
655.4 \\
655.4 \\
655.2\end{array}$ & $\begin{array}{l}665.0 \\
664.7 \\
664.5 \\
664.8\end{array}$ & $\begin{array}{l}673.2 \\
672.8 \\
673.4 \\
672.8\end{array}$ & $\begin{array}{l}689.6 \\
689.9 \\
690.7 \\
690.2\end{array}$ & $\begin{array}{l}710.9 \\
709.3 \\
708.6 \\
709.1\end{array}$ & 1 \\
\hline
\end{tabular}


TABLE A1 (continued)

\begin{tabular}{|c|c|c|c|c|c|c|c|c|c|}
\hline \multirow[b]{2}{*}{ Run } & \multicolumn{8}{|c|}{ Signal factor levels } & \multirow[b]{2}{*}{$X_{N}$} \\
\hline & 650 & 700 & 750 & 800 & 850 & 900 & 950 & 1,000 & \\
\hline \multirow[t]{4}{*}{1} & 639.7 & 642.3 & 645.5 & 653.9 & 666.6 & 672.1 & 692.2 & 711.6 & \multirow[t]{4}{*}{-1} \\
\hline & 640.5 & 641.7 & 644.8 & 655.1 & 665.8 & 670.8 & 690.6 & 710.8 & \\
\hline & 636.2 & 643.6 & 646.1 & 654.7 & 667.1 & 673.3 & 689.7 & 711.1 & \\
\hline & 637.2 & 644.0 & 644.3 & 654.2 & 665.4 & 671.1 & 689.8 & 710.5 & \\
\hline \multirow[t]{4}{*}{2} & 634.4 & 639.9 & 642.6 & 650.2 & 659.9 & 666.8 & 678.4 & 708.3 & \multirow[t]{4}{*}{-1} \\
\hline & 632.9 & 640.8 & 640.4 & 651.6 & 660.3 & 660.3 & 682.6 & 710.1 & \\
\hline & 633.7 & 641.1 & 643.1 & 650.9 & 657.9 & 659.8 & 681.8 & 707.7 & \\
\hline & 635.8 & 642.4 & 641.9 & 653.2 & 662.1 & 661.5 & 683.2 & 706.6 & \\
\hline \multirow[t]{4}{*}{3} & 640.2 & 646.1 & 647.2 & 655.5 & 666.2 & 671.0 & 688.6 & 708.9 & \multirow[t]{4}{*}{-1} \\
\hline & 638.1 & 644.4 & 646.0 & 654.0 & 667.3 & 673.6 & 687.5 & 710.0 & \\
\hline & 637.3 & 644.4 & 647.5 & 653.8 & 669.1 & 672.4 & 691.0 & 711.3 & \\
\hline & 639.1 & 641.2 & 644.3 & 652.8 & 664.7 & 672.2 & 693.1 & 708.4 & \\
\hline \multirow[t]{4}{*}{4} & 641.1 & 644.5 & 647.2 & 652.0 & 665.3 & 669.2 & 688.7 & 709.8 & \multirow[t]{4}{*}{-1} \\
\hline & 642.1 & 647.3 & 644.8 & 654.6 & 661.0 & 671.1 & 690.4 & 710.1 & \\
\hline & 642.0 & 642.8 & 646.0 & 653.8 & 659.7 & 670.1 & 686.3 & 707.7 & \\
\hline & 641.8 & 643.9 & 646.3 & 651.7 & 662.4 & 671.1 & 685.8 & 706.4 & \\
\hline \multirow[t]{4}{*}{5} & 640.8 & 644.7 & 647.6 & 652.3 & 661.1 & 673.0 & 685.7 & 706.4 & \multirow[t]{4}{*}{-1} \\
\hline & 641.1 & 645.3 & 646.8 & 654.5 & 662.8 & 673.2 & 686.7 & 707.7 & \\
\hline & 641.2 & 644.6 & 647.3 & 653.9 & 659.2 & 672.5 & 686.2 & 706.9 & \\
\hline & 641.6 & 645.0 & 647.5 & 653.6 & 659.9 & 673.7 & 686.1 & 706.3 & \\
\hline \multirow[t]{4}{*}{6} & 650.4 & 655.4 & 659.7 & 665.8 & 671.0 & 677.7 & 695.6 & 716.5 & \multirow[t]{4}{*}{-1} \\
\hline & 650.8 & 655.0 & 660.2 & 665.9 & 670.8 & 677.5 & 696.8 & 717.0 & \\
\hline & 651.2 & 654.6 & 660.3 & 665.9 & 671.2 & 678.2 & 694.3 & 718.3 & \\
\hline & 650.7 & 654.9 & 659.3 & 666.4 & 670.5 & 677.8 & 696.1 & 717.6 & \\
\hline \multirow[t]{4}{*}{7} & 639.6 & 643.8 & 648.2 & 655.7 & 665.2 & 674.8 & 691.7 & 710.1 & \multirow[t]{4}{*}{-1} \\
\hline & 639.4 & 644.2 & 647.3 & 656.0 & 664.8 & 675.3 & 691.4 & 711.4 & \\
\hline & 639.9 & 644.1 & 647.2 & 655.5 & 664.3 & 675.0 & 691.8 & 710.3 & \\
\hline & 640.0 & 644.4 & 647.8 & 656.2 & 663.9 & 675.1 & 692.3 & 711.1 & \\
\hline \multirow[t]{4}{*}{8} & 636.5 & 641.8 & 645.2 & 653.8 & 662.8 & 671.8 & 689.4 & 709.7 & \multirow[t]{4}{*}{-1} \\
\hline & 636.2 & 640.6 & 646.1 & 653.9 & 662.3 & 671.6 & 689.1 & 709.6 & \\
\hline & 635.7 & 640.5 & 645.5 & 653.9 & 662.1 & 671.6 & 689.6 & 709.7 & \\
\hline & 636.1 & 640.3 & 645.0 & 653.6 & 662.4 & 671.6 & 689.3 & 709.3 & \\
\hline
\end{tabular}

\section{ACKNOWLEDGMENTS}

This research was supported by the Natural Sciences and Research Council of Canada, the University of Auckland Research Committee (for the first author) and AFOSR/ARPA Grant F49620-95-1-0524 (for the second author).

\section{REFERENCES}

BoceK, P. and NovaK, J. (1970). Statistical processing of calibration data in quantitative GC. Journal of Chromatography $\mathbf{5 1}$ $375-383$.

Box, G. (1988). Signal-to-noise ratios, performance criteria, and transformations. Technometrics 30 1-40.

Box, G. and JonEs, S. (1992). Split-plot designs for robust product experimentation. J. Appl. Statist. 19 3-26.

Box, G. E. P., Hunter, W. G. and Hunter, J. S. (1978). Statistics for Experimenters. Wiley, New York.

DeMATES, J. (1990). Dynamic analysis of injection molding using Taguchi methods. In Eighth Symposium on Taguchi Methods 313-331. American Supplier Institute, Dearborn, MI.

GunTER, B. (1988). Discussion of signal-to-noise ratios, performance criteria, and transformations by G. E. P. Box. Technometrics 30 32-35.

Leon, R. V., Shoemaker, A. C. and Kacker R. N. (1987). Per- formance measures independent of adjustment: an explanation and extension of Taguchi's signal to noise ratio. Technometrics 29 253-285.

MANDEL, J. (1964). The Statistical Analysis of Experimental Data. Interscience, New York.

MilleR, A. E. and WU C. F. J. (1991). Improving a calibration system through designed experiments. Research Report 9106, Institute for Improvement in Quality and Productivity, Univ. Waterloo.

NAIR, V. N., EDITOR (1992). Taguchi's parameter design: a panel discussion. Technometrics 34 127-161.

Phadke, M. S. (1989). Quality Engineering Using Robust Design. Prentice-Hall, Englewood Cliffs, NJ.

Pignatiello J. J. JR. and RAMberG J. S. (1985). Discussion of "Off-line quality control, parameter design, and the Taguchi method" by R. N. Kacker. Journal of Quality Technology 17 198-206.

Shoemaker, A. C., Tsui, K. L. and Wu, C. F. J. (1991). Economical experimentation methods for robust design. Technometrics 33 415-427.

TAGUCHI, G. (1987). System of Experimental Design. Unipub/ Kraus International Publications, White Plains, NY.

WELCH, W. J., YU, T. K., KANG, S. M. and SACKS, J. (1990). Computer experiments for quality control by parameter design. Journal of Quality Technology 22 15-22.

Yano, H. (1991). Metrological Control: Industrial Measurement Management. Asian Productivity Organization, Tokyo. 